\title{
Selection of Feature for Epilepsy Seizer Detection Using EEG
}

\author{
Manisha Chandani ${ }^{1}$, Arun Kumar ${ }^{2}$ \\ Department of Electronics \& Telecommunication, Bhilai Institute of Technology, Durg, India
}

Email address:

chandani.manisha09@gmail.com(M.Chandani),arun.kumar@bitdurg.ac.in(A.Kumar)

\section{To cite this article:}

Manisha Chandani, Arun Kumar. Selection of Feature for Epilepsy Seizer Detection Using EEG. International Journal of Neurosurgery. Vol. 2, No. 1, 2018, pp. 1-7. doi: 10.11648/j.ijn.20180201.11

Received: January 8, 2018; Accepted: March 23, 2018; Published: April 20, 2018

\begin{abstract}
The study of the electrical signals produced by neural activities of human brain is called Electroencephalography. Epilepsy is one of the most common neurological diseases and the most common neurological chronic disease in childhood. Electroencephalography (EEG) still remains one of the most useful and effective tools in understanding and treatment of epilepsy. EEG signal when decomposed into frequency subbands, gives us several statistical features in each band. Some of these features that may be employed for detection of epilepsy are explored in this paper.
\end{abstract}

Keywords: Electroencephalography (EEGs), Epileptic, Seizure

\section{Introduction}

Epilepsy is a neurological disorder due to excessive neuronal activity in the brain characterized by recurrent brain malfunction. Epilepsy can be assessed by the EEG i.e. the electrical activity of brain. It is highly non-linear and nonstationary, and therefore is difficult to characterize and interpret. The signals are normally presented in the time domain, but many new EEG machines are capable of applying simple signal processing tools such as the Fourier transform to perform frequency analysis. There have been many algorithms developed so far for processing EEG signals. The operations include, but are not limited to, timedomain analysis, frequency-domain analysis, and spatialdomain analysis. For past many years there are various features that have been extracted by the researchers for the purpose of classification of EEG signal to be epileptic or not on the basis of the those features [1-4].

\section{Data Selection and Recording}

In proposed work publicly available data ia used described in [5]. The complete data set consists of five sets (denoted AE) each containing 100 single-channel EEG segments. These segments were selected and cut out from continuous multichannel EEG recordings after visual inspection for artefacts, e.g., due to muscle activity or eye movements.

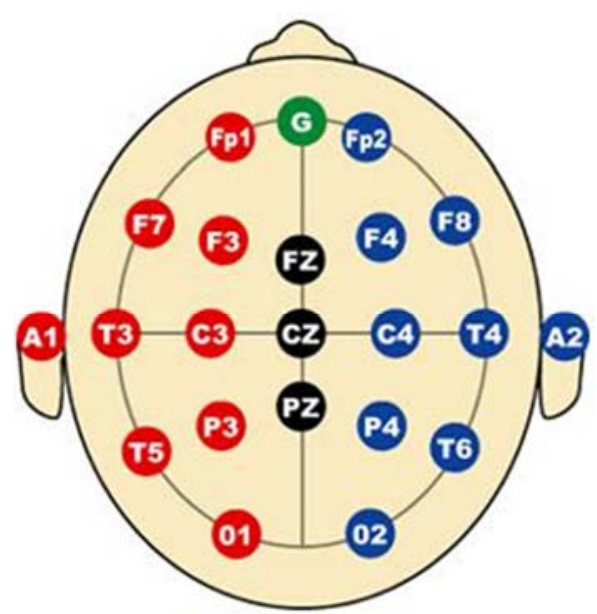

Figure 1. The 10-20 international system of electrode placement images of normal and abnormal cases.

Sets A and B consisted of segments taken from surface EEG recordings that were carried out on five healthy volunteers using a standardised electrode placement scheme.

Volunteers were relaxed in associate awake-state with eyes open (A) and eyes closed (B), severally. Sets C, D, and E originated from graph archive of pre-surgical identification. EEGs from 5 patients were hand-picked, all of whom had achieved complete seizure management when surgical operation of one of the hippocampal formations, that was 
thus properly diagnosed to be the epileptogenic zone. Segments in set D were recorded from among the epileptogenic zone, and folks in set $\mathrm{C}$ from the hippocampal formation of the opposite hemisphere of the brain. Whereas sets $\mathrm{C}$ and $\mathrm{D}$ contained alone activity measured throughout seizure free intervals, set $\mathrm{E}$ alone contained seizure activity.

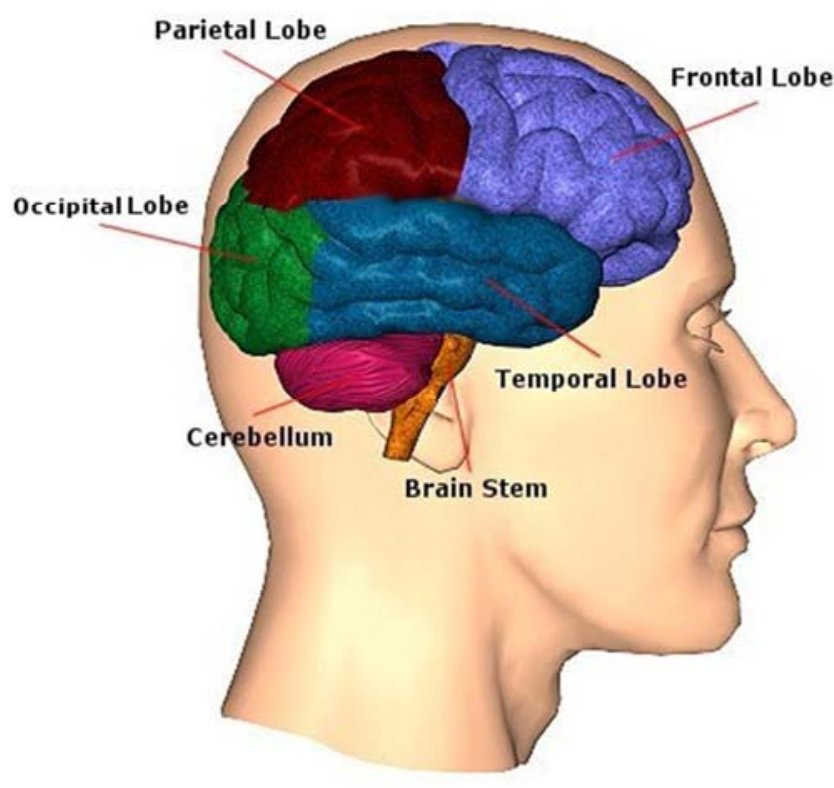

Figure 2. Human brain structur.
The electrodes were set as shown in figure. 1. Therefore, the electrodes named as: FP1, FP2, F3, F4, C3, C4, P3, P4, F7, F8, T1, T2, T3, T5, T6, O1, O2, F2, P2. The frontal lobe, temporal lobe, parietal lobe, central lobe, and occipital lobe were named F, T, P, C, and O respectively [6]

The Figure 2 describe the anatomy of the brain with different regions [7].

The cerebrum is the largest part and is responsible for initiation of movement, coordination of movement, sensing temperature, touch, vision, hearing, judgment, reasoning, problem solving, emotions and learning. Cerebrum is divided into four lobes. They are frontal lobe, occipital lobe, parietal lobe \& temporal lobe. Here segments were hand-picked from all recording sites exhibiting ictic activity.

All encephalogram (EEG) signals were recorded with constant 128-channel electronic equipment system, victimisation a mean common reference.

The info were digitised at 173.61 samples per second victimisation twelve bit resolution. Band pass filter settings were $0.53-40 \mathrm{cps}(12 \mathrm{~dB} / \mathrm{oct})$. In this study, a tendency to used 2 dataset (A and $\mathrm{E}$ ) of the whole dataset. The signals which have been taken as input for the analysis purpose. Figure. 3, 4, 5 show healthy, convulsive and epileptic signals. The signal overlap healthy and epileptic shown in Figure. 6. In processing medical signals, it is vitally important to minimize existing noises and artifacts in order that they have the minimum effect on the feature extraction stage.

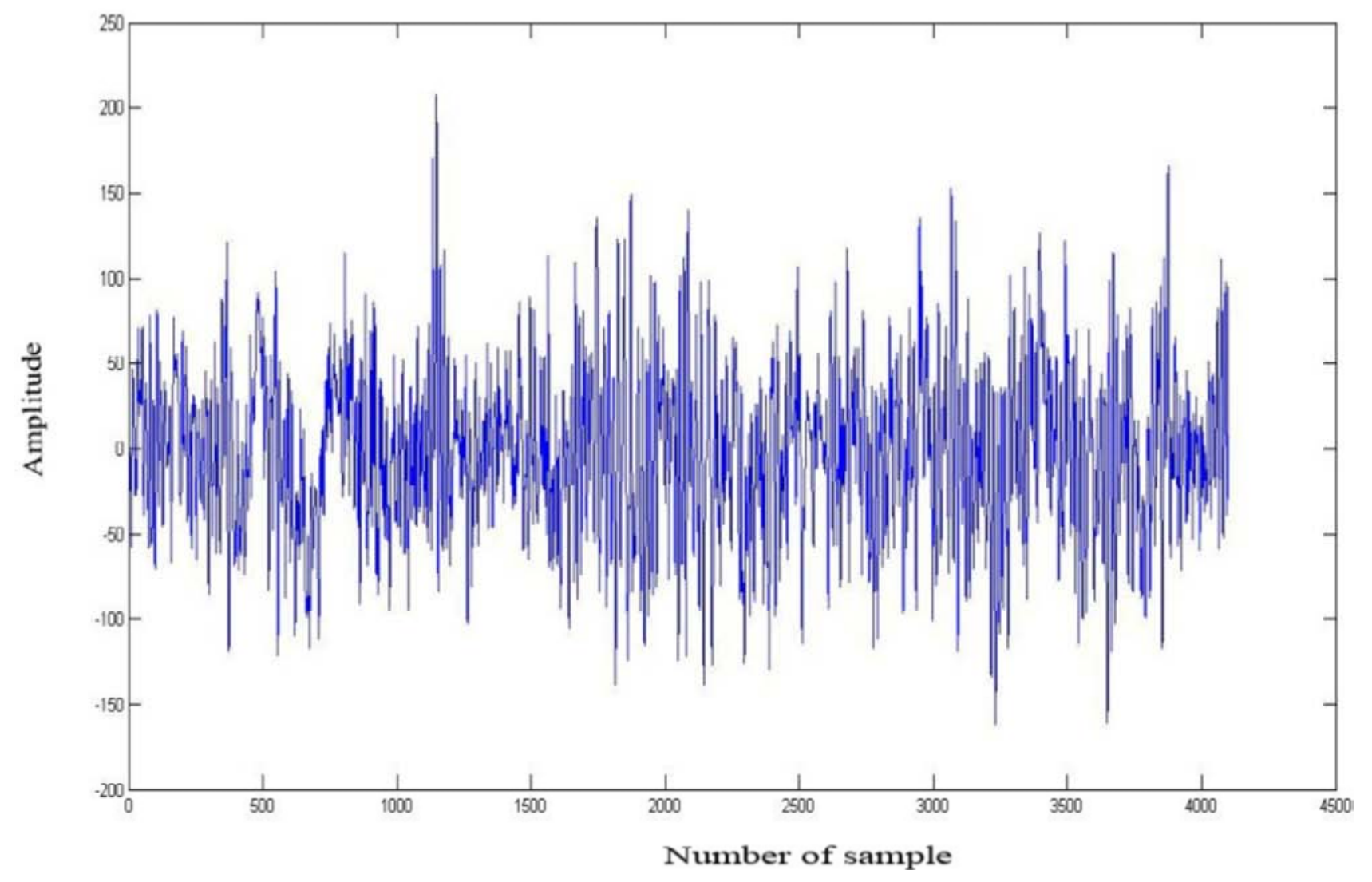

Figure 3. An example of healthy signals. 


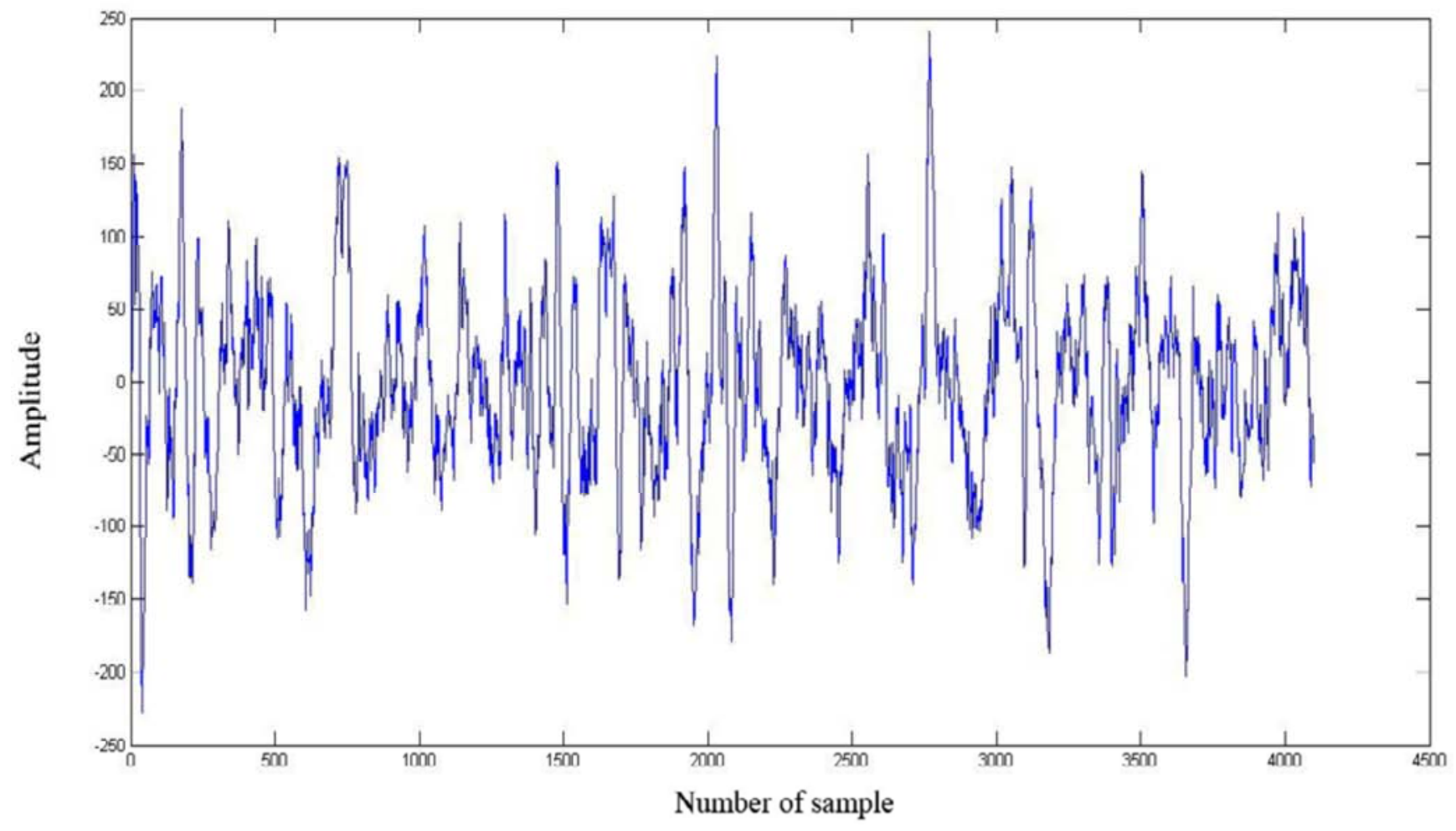

Figure. 4 An example of convulsive signals.

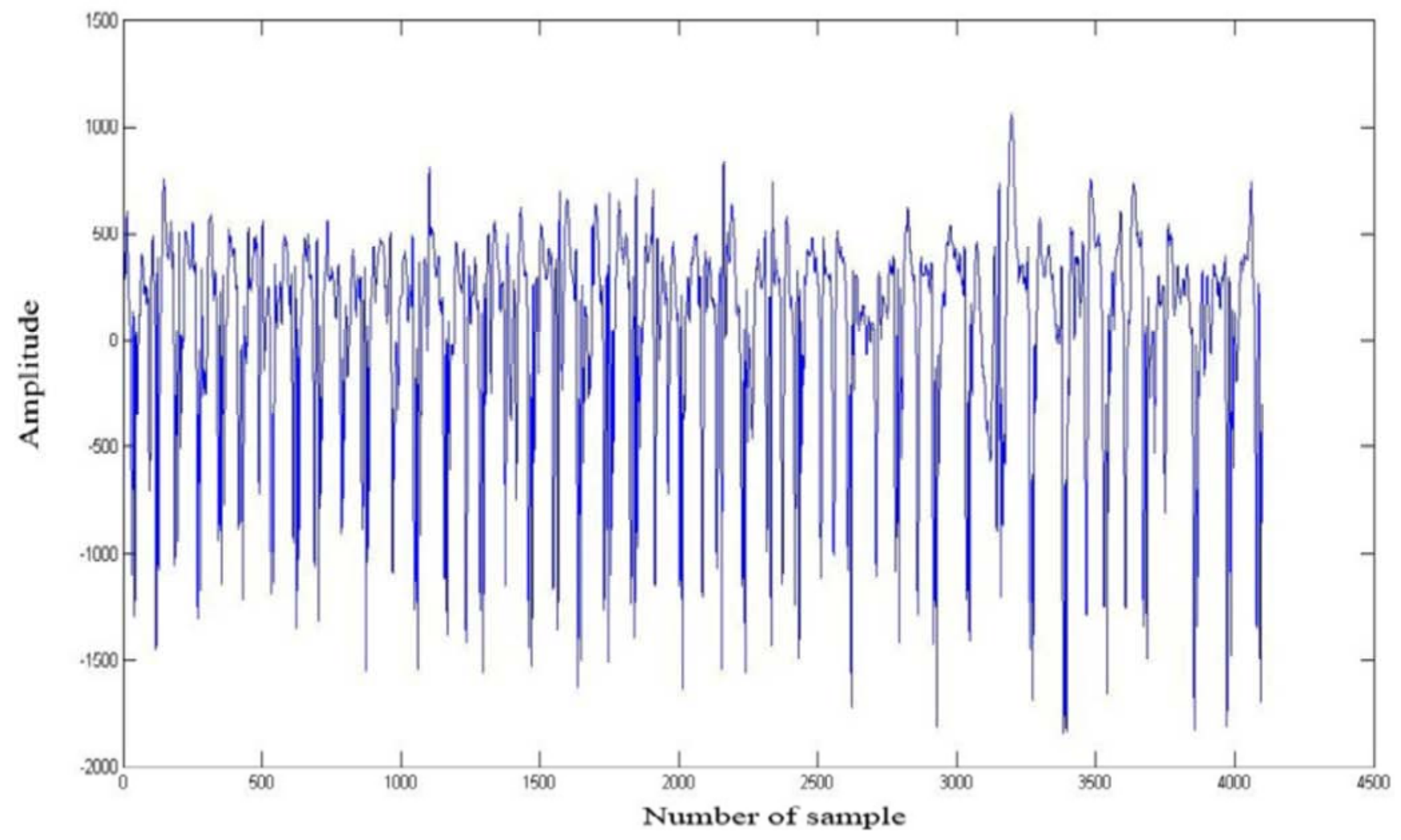

Figure 5. An example of epileptic signals. 


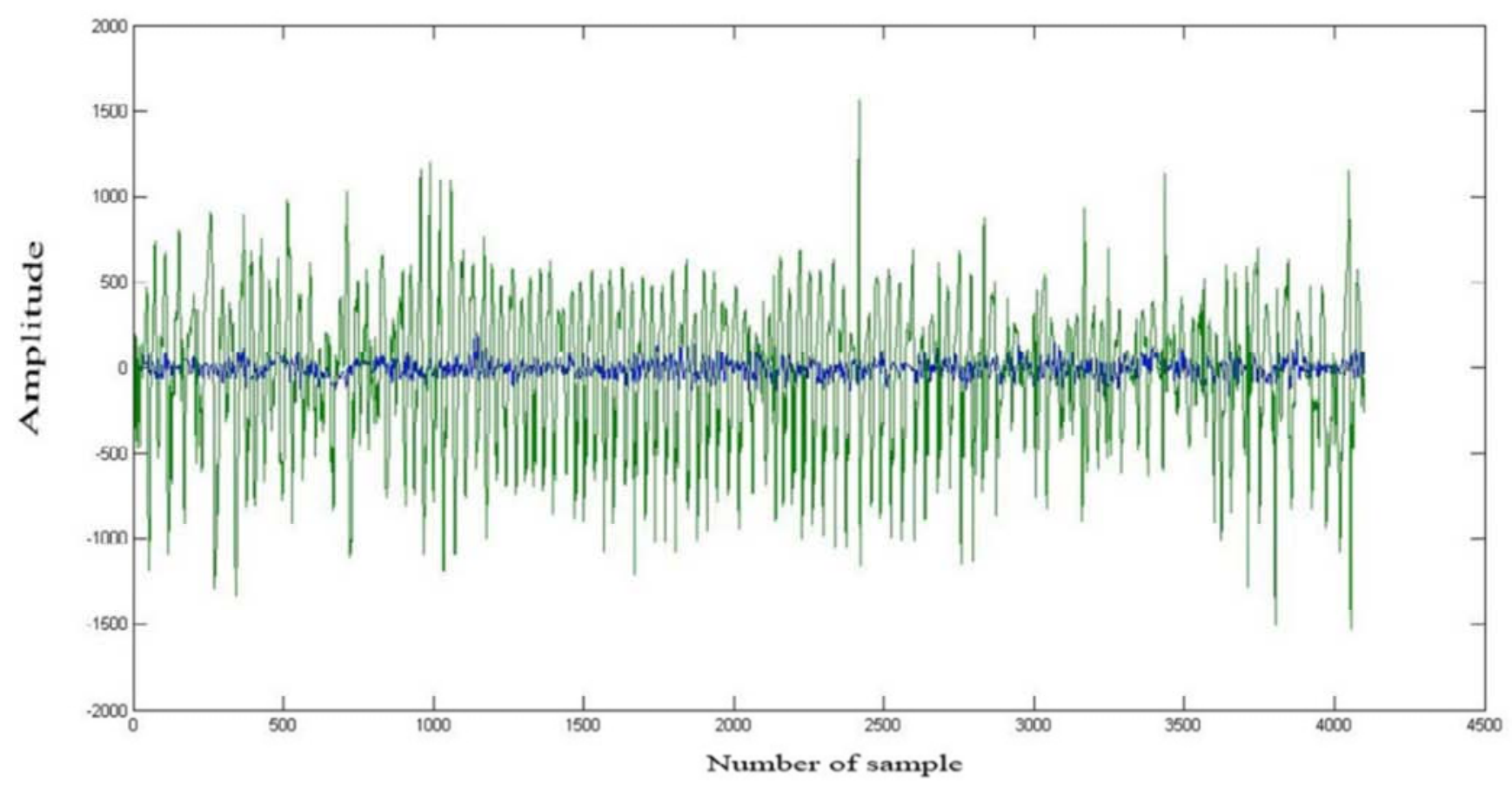

Figure 6. Healthy and epileptic signals overlap rate.

\section{Problem Identification}

For identifying the epileptic and non-epileptic EEG, it is required to examine subject for selected features extracted from the subbands of EEG. It is probable that some of the features may have non-overlapping range and useful to achieve highest frequency. Those features are needed to be identified for detection of epilepsy.

\section{Methodology}

The subband decomposition of the EEG signal explicitly explained in [8] is applied on the sets of epileptic and nonepileptic data of 50 subjects each. Each set is composed of 4096 samples at sampling frequency of $173.6 \mathrm{~Hz}$. In this study, the discrete wavelet transform is used as a primary computational tool for extracting features of the epileptic EEG signals at different resolutions. Decomposition of Epileptic and Non-Epileptic data into Delta, Theta, Alpha, Beta, Gamma subbands are shown in figure 1 and Figure 2 respectively. It is apparent from figures that the amplitude of gross epileptic signal is considerably higher than non-epileptic one. Also the amplitudes of subbands are significantly high in case of epileptic data, especially in gamma subband.

The statistical parameters all the extracted features constitute the combined feature index $(\mathrm{CFI})=(\mathrm{F} 1, \mathrm{~F} 2, \mathrm{~F} 3$, $\mathrm{F} 4, \mathrm{~F} 5 \ldots . . . \mathrm{Fn}$ ), which is presented as an input to the system. The features used in evaluating the performance of the scheme are mean, standard deviation, median, entropy, kurtosis and skewness were calculated at each decomposition level starting from 01 to 04 for the normal and epilepsy categories of signals [8].

Mean $\mu$

Mean are fundamental statistical attributes of a time series the arithmetic mean of a time seriesis the average expected value of that time series. In some cases, the mean value of a time series can be the operating point or working point of a physical system that generates the time series.

$$
\begin{gathered}
\mu=\frac{1}{N} \sum_{i=1}^{N} A_{i} \\
i=1,2,3, \ldots \ldots \ldots, N .
\end{gathered}
$$

The mean indicated by $\mu$. The Value in the signal $\mathrm{X}$, byletting the index, $\mathrm{i}$, run from 0 to 1 . Then finish the calculation by dividing the sum by $\mathrm{N}$. This is identical to the equation: $\mu=\left(X_{0}+X_{1}+X_{2}+\ldots+X_{N-1}\right) / \mathrm{N}$.

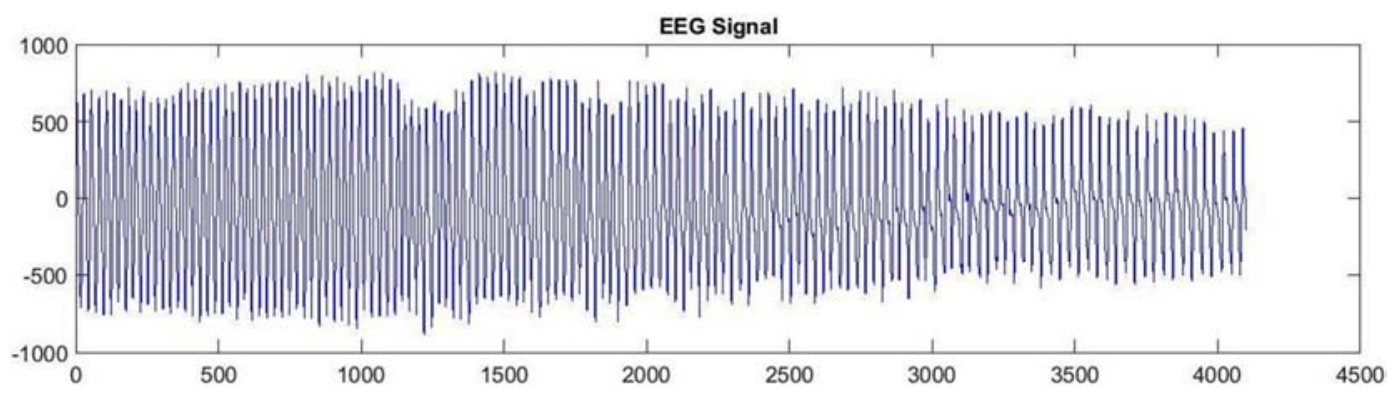



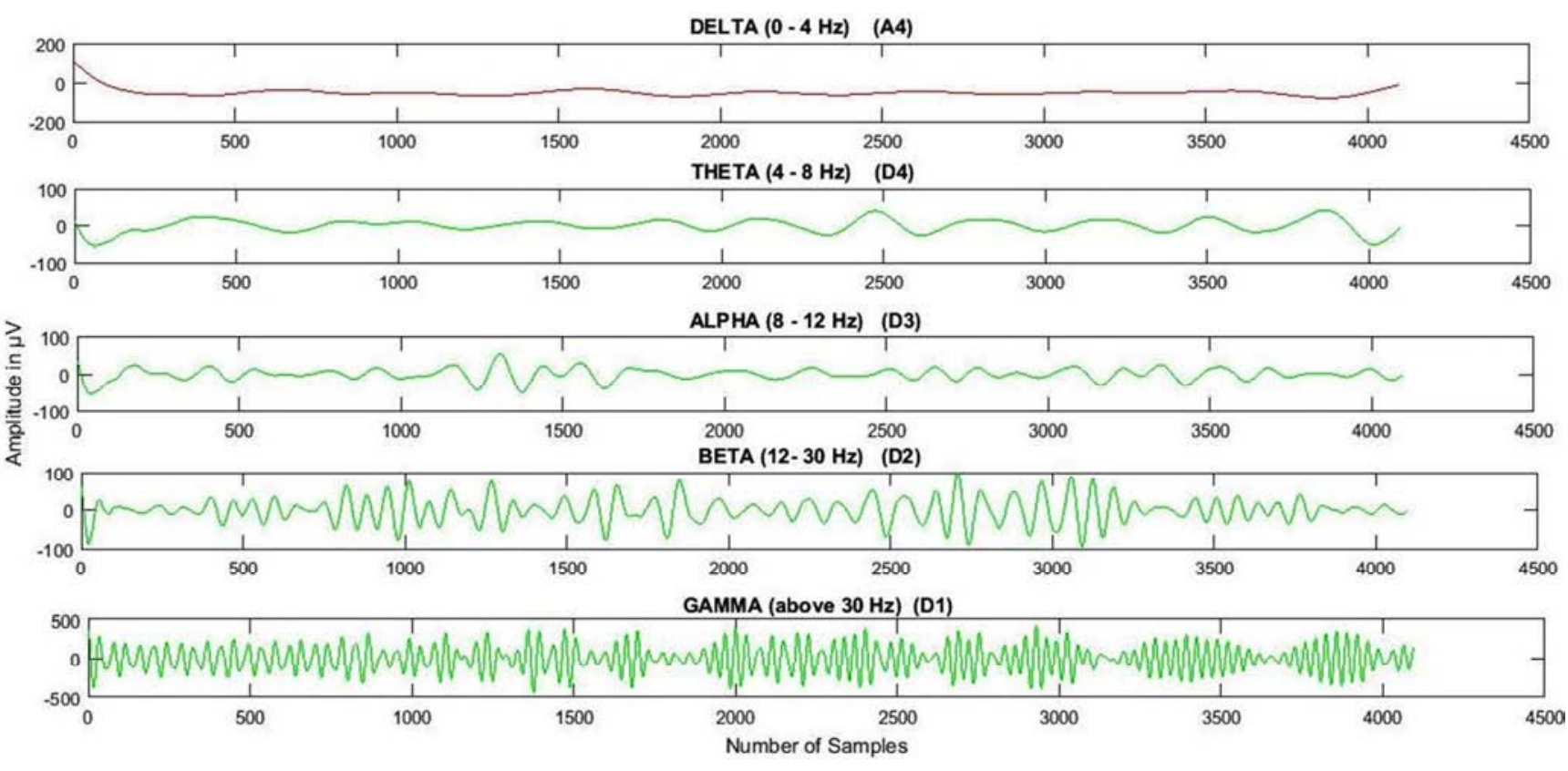

Figure 7. Epileptic EEG signal with delta, theta, alpha, beta and gamma subband decomposition.
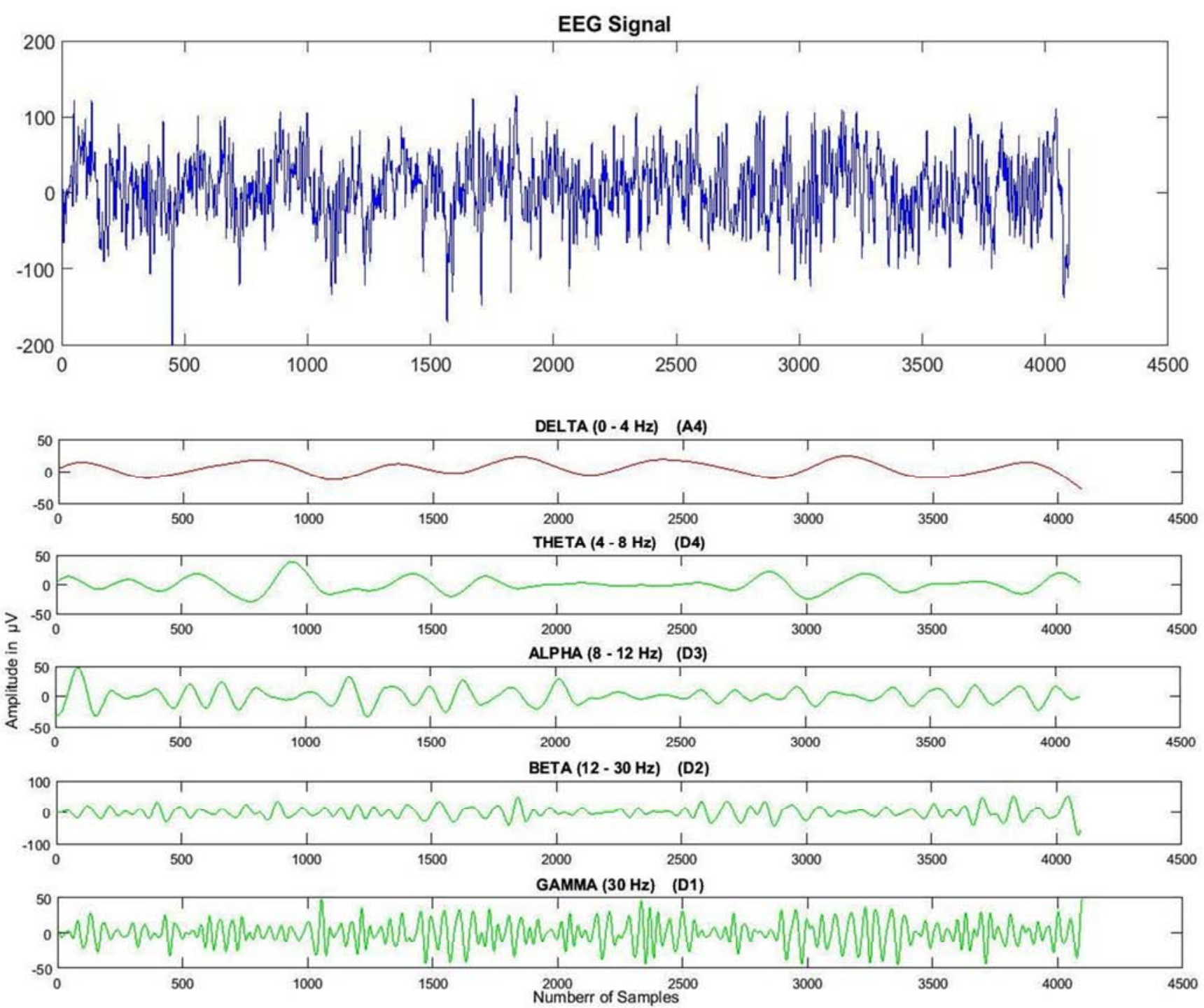

Figure 8. Non-epileptic EEG signal with delta, theta, alpha, beta and gamma subband composition. 
Standard Deviation $\sigma$

The standard deviation is similar to the average deviation except the averaging is done with power instead of amplitude. This is achieved by suaring each of the deviation before taking the average. To finish the square root is taken to compensate for the intial squaring. In equation from the standard deviation is calculation:

$$
\begin{gathered}
\sigma=\sqrt{\frac{1}{N} \sum_{i=1}^{N}\left(x_{i}-\mu\right)^{2}} \\
i=1,2,3, \ldots \ldots . ., N .
\end{gathered}
$$

$\mathrm{X}$ is signal with mean $\mu, \mathrm{N}$ is the number of sample and $\sigma$ is standard deviation.

Kurtosis k

The kurtosis is higher-order statistical attributes of a time series. Kurtosis measures the peakedness of the probability density function (PDF) of a time series. A kurtosis value close to three indicates a Gaussian-like peakedness. PDFs with relatively sharp peaks have kurtosis greater than three. PDFs with relatively flat peaks have kurtosis less than three.

$$
k=\frac{E(x-\mu)^{4}}{\sigma^{4}}
$$

Where $\mathrm{X}$ is a signal, $\mu$ is the mean of $x, \sigma$ is the standard deviation of $X$, and $E(t)$ represented the expected value of the quantity $t$ Kurtosis computes a sample version of this population value.

Skewness $\mathrm{S}$

The skewness are higher-order statistical attributes of a time series. Skewness indicates the symmetry of the probability density function (PDF) of the amplitude of a time series. A time series with an equal number of large and small amplitude values has a skewness of zero. A time series with many small values and few large values is positively skewed and the skewness value is positive. A time series with many large values and few small values is negatively skewed and the skewness value is negative.

$$
S=\frac{E(x-\mu)^{4}}{\sigma^{4}}
$$

Where $\mu$ is the mean of signal $x, \sigma$ is the standard deviation of $\mathrm{x}$, and $\mathrm{E}(\mathrm{t})$ represented the expected value of the quantity $\mathrm{t}$ Skewness computes a sample version of this population value.

\section{Entropy E}

Entropy is a numerical measure of the randomness of a signal. Entropy can act as a feature and used to analyze psychological time series data such as EEG data. The Entropy can thus be calculated as:

$$
\begin{gathered}
E(s)=\sum_{i} E\left(s_{i}\right) \\
i=1,2,3, \ldots \ldots ., N .
\end{gathered}
$$

The E must be the an additive cost function such that $\mathrm{E}(0)$ $=0$. Entropy is the statistical descriptor of the variability within the EEG signal and is a strong feature for epilepsy detection.

Median M

The median is a simple measure of central tendency. To find the median, we arrange the observations in order from smallest to largest value. If there are an odd number of observations, the median is the middle value. If there is an even number of observations, the median is the average of the two middle values

$$
\bar{X}=\frac{\sum_{i=1}^{n} X_{i}}{n}
$$

$\mathrm{X}$ refers to the entire set of the numbers. Median is more robust than arithmetic mean and geometric mean if the raw data does not contain significant outliers.

\section{Rest and Conclusion}

These 30 parameters analysed for 50 non-epileptic and 50 epileptic cases taken from database [8], it is found that that these 30 parameters are highly suitable for the detection of epilepsy with $100 \%$ accuracy [8]. These parameters are illustrated in the Table 1 . These features can be significantly used for detection of epilepsy.

Table 1. Nomenclature of Feature Subbands.

\begin{tabular}{llllll}
\hline Sabband Features & Gamma & BETA & ALPHA & THETA & DELTA \\
\hline Mean & M1 & M2 & M3 & M4 & M5 \\
Standard deviation & S1 & S2 & S3 & S4 & S5 \\
Median & Md1 & Md2 & Md3 & Md4 & Md5 \\
Entropy & E1 & E2 & E3 & E4 & E5 \\
Kurtosis & K1 & K2 & K3 & K4 & K5 \\
Skewness & Y1 & Y2 & Y3 & Y4 & Y5 \\
\hline
\end{tabular}

\section{Future Scope}

There are several different features which have different significant. Yet some different features needs to be compare or use to achieve the highest or full accuracy.

\section{References}

[1] Abdulhamit Subasi (2007). "EEG Signal Classification Using Wavelet Feature Extraction and a Mixture of Expert Model", Expert Systems with Applications 32, pp. no 1084-1093.

[2] N. Kannathal, Min Lim Choo, U. Rajendra Acharya and P. K. Sadasivan (2005). "Entropies for Detection of Epilepsy in EEG", Computer Methods and Programs in Biomedicine 80, pp. no 187-194.

[3] Tapan Gandhi, Bijay Ketan Panigrahi and Sneh Anand (2011). "A Comparative Study of Wavelet Families for EEG Signal Classification", Neurocomputing 74, pp no 3051-3057.

[4] R. K. Chaurasiya, N. D. Londhe and S. Ghosh (2015). "Statistical Wavelet Features, PCA, and SVM Based Approach for EEG Signals Classification" IJECEECE, Vol: 9, pp. 182-186. 
[5] R. G. Andrezejak, K. Lehnertz \& F. Morman, (2001). Indication of nonlinear deterministic and finite-dimensional structures in time series of brain electrical activity: dependence on recording region and brain state. Phys. Rev. Ed-64 (6)-061907.

[6] Durka P. J. (2004). Adaptive time-frequency parametrization of epileptic spikes. Physical Review E; 69:051914.

[7] Kumari Pinki \& AbhishekVaish (2015). Brainwave based user identification system: A pilot study in robotics environment. Robotics and Autonomous Systems 65, pp. 15-23.

[8] Arun Kumar and Manisha Chandani (2017). "Analysis of EEG Physiological Signal for The Detection of Epileptic Seizure " i-manager's Journal on Pattern Recognation, Vol. 4, pp. 1-9.

[9] Subasi A (2007). "EEG signal classification using wavelet feature extraction and a mixture of expert model," Expert Syst Appl., vol. 32 (4), pp. 1084-1093.

[10] Meenakshi, Dr. R. K Singh, Prof. A. K Singh (2014). "Frequency Analysis of Healthy \& Epileptic Seizure in EEG using Fast Fourier Transform" International Journal of Engineering Research and General Science Volume 2, Issue 4, pp- 683-691.
[11] Welch P D (1967). "The use of fast fourier transform for the estimation of power spectra: a method based on time averaging over short, modified periodograms," IEEE Trans Audio Electroacoust., vol. AU-15, pp. 70-73.

[12] Arun Kumar and Manisha Chandani (2017)“Classification of EEG Physiological Signal for the Detection of Epileptic Seizure by Using DWT Feature Extraction and Neural Network " International Journal of Neurologic Physsical Therapy Vol. 3., pp- 38-43.

[13] Abdulhamit Subasi (2007). "EEG Signal Classification Using Wavelet Feature Extraction and a Mixture of Expert Model", Expert Systems with Applications 32, pp. no 1084-1093.

[14] N. Kannathal, Min Lim Choo, U. Rajendra Acharya and P. K. Sadasivan (2005). "Entropies for Detection of Epilepsy in EEG", Computer Methods and Programs in Biomedicine 80, pp. no 187-194.

[15] Tapan Gandhi, Bijay Ketan Panigrahi and Sneh Anand (2011). "A Comparative Study of Wavelet Families for EEG Signal Classification", Neurocomputing 74, pp no 3051-3057. 